
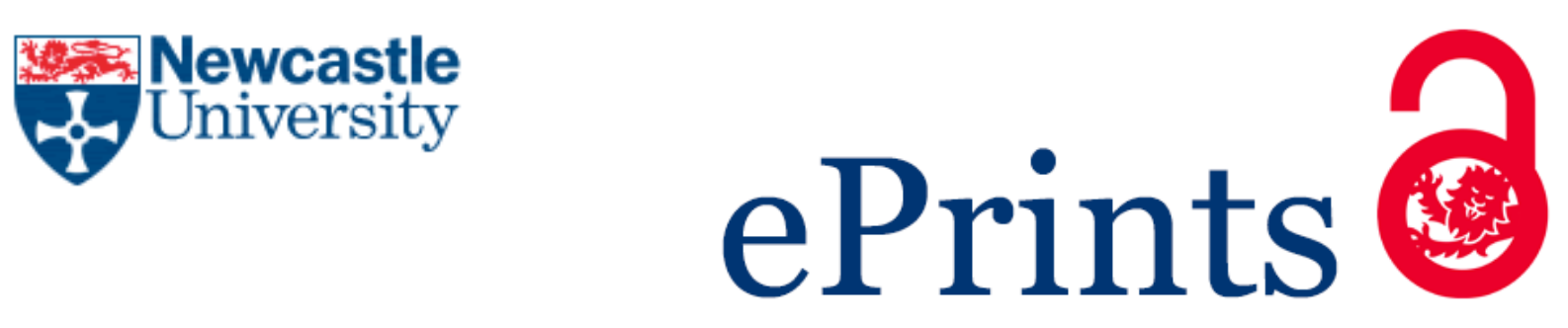

Sokolov D, Khomenko V, Mokhov A, Yakovlev A, Lloyd D. Design and Verification of Speed-Independent Multiphase Buck Controller. In: 21st IEEE International Symposium on Asynchronous Circuits and Systems (ASYNC'15). 2015, Mountain View, Silicon Valley, California, USA: IEEE Computing Society Press.

\title{
Copyright:
}

(C) 2015 IEEE. Personal use of this material is permitted. Permission from IEEE must be obtained for all other uses, in any current or future media, including reprinting/republishing this material for advertising or promotional purposes, creating new collective works, for resale or redistribution to servers or lists, or reuse of any copyrighted component of this work in other works.

DOI link to article:

http://dx.doi.org/10.1109/ASYNC.2015.14

Date deposited:

$21 / 07 / 2015$ 


\section{Design and Verification of Speed-Independent \\ Multiphase Buck Controller}

Danil Sokolov ${ }^{1}$, Victor Khomenko ${ }^{1}$, Andrey Mokhov ${ }^{1}$, Alex Yakovlev ${ }^{1}$, David Lloyd ${ }^{2}$

${ }^{1}$ Newcastle University, UK; ${ }^{2}$ Dialog Semiconductor, UK 


\section{Motivation}

- Efficient implementation of power converters is paramount

- Extending the battery life of mobile gadgets

- Reducing the energy bill for PCs and data centres $(5+3 \%$ of global electricity production)

- Need for responsive and reliable control circuitry - little digital

- Millions of control decisions per second for years

- An incorrect decision may permanently damage the circuit

- Poor EDA support

- Synthesis is optimised for data processing - big digital

- Ad hoc solutions are prone to errors and cannot be verified 


\section{Basic buck converter: Schematic}

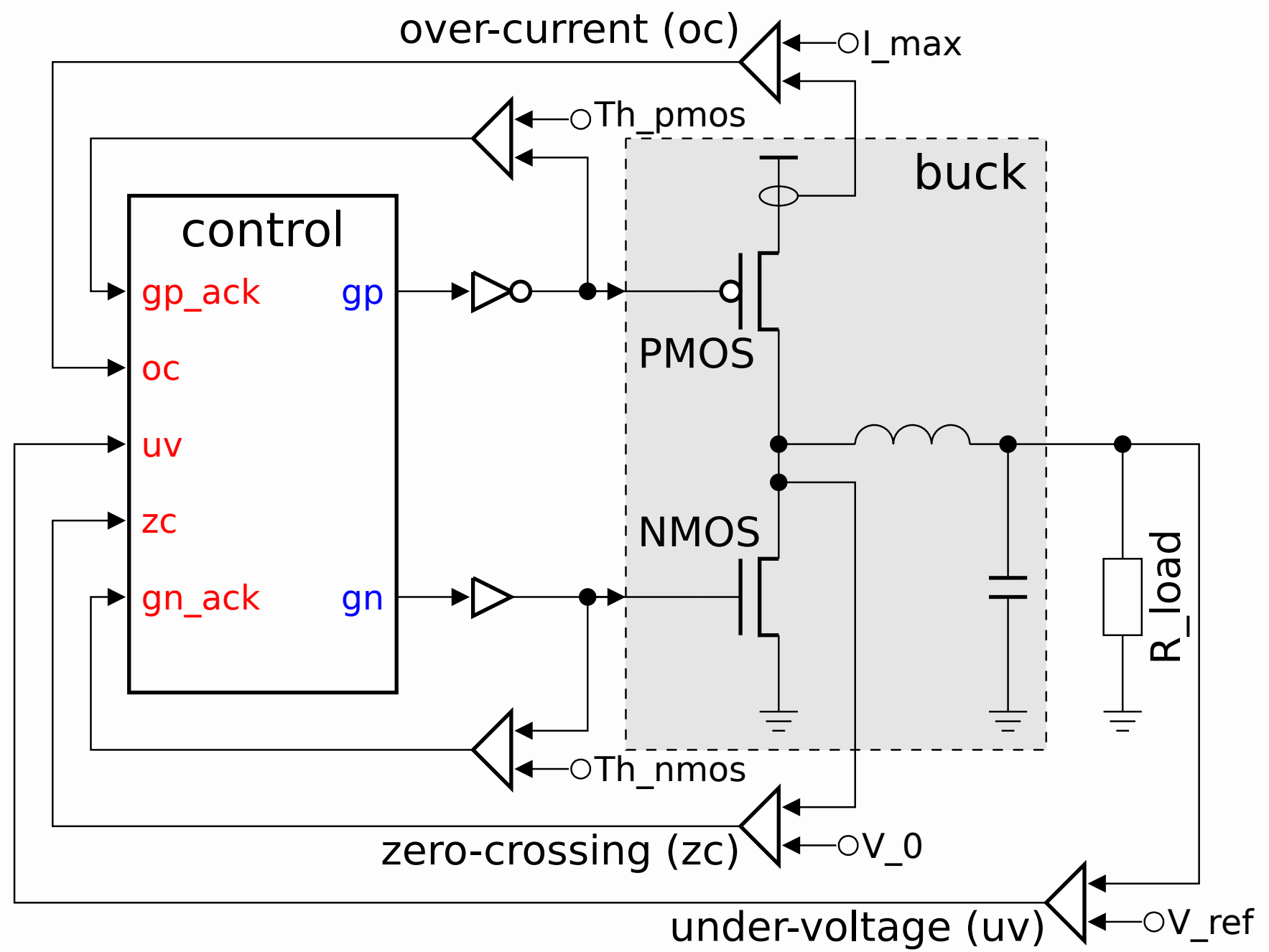

- In the textbook buck a diode is used instead of NMOS transistor 


\section{Basic buck converter: Informal specification}

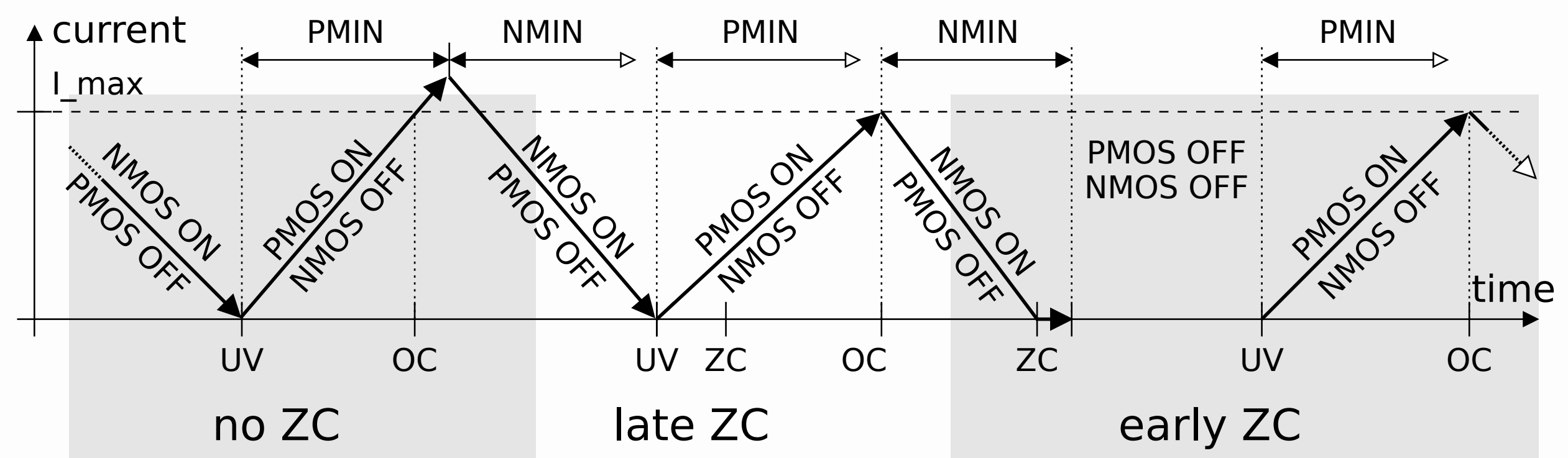

- no ZC - under-voltage without zero-crossing

- late ZC - under-voltage before zero-crossing

- early ZC - under-voltage after zero-crossing 


\section{Multiphase buck converter: Schematic}

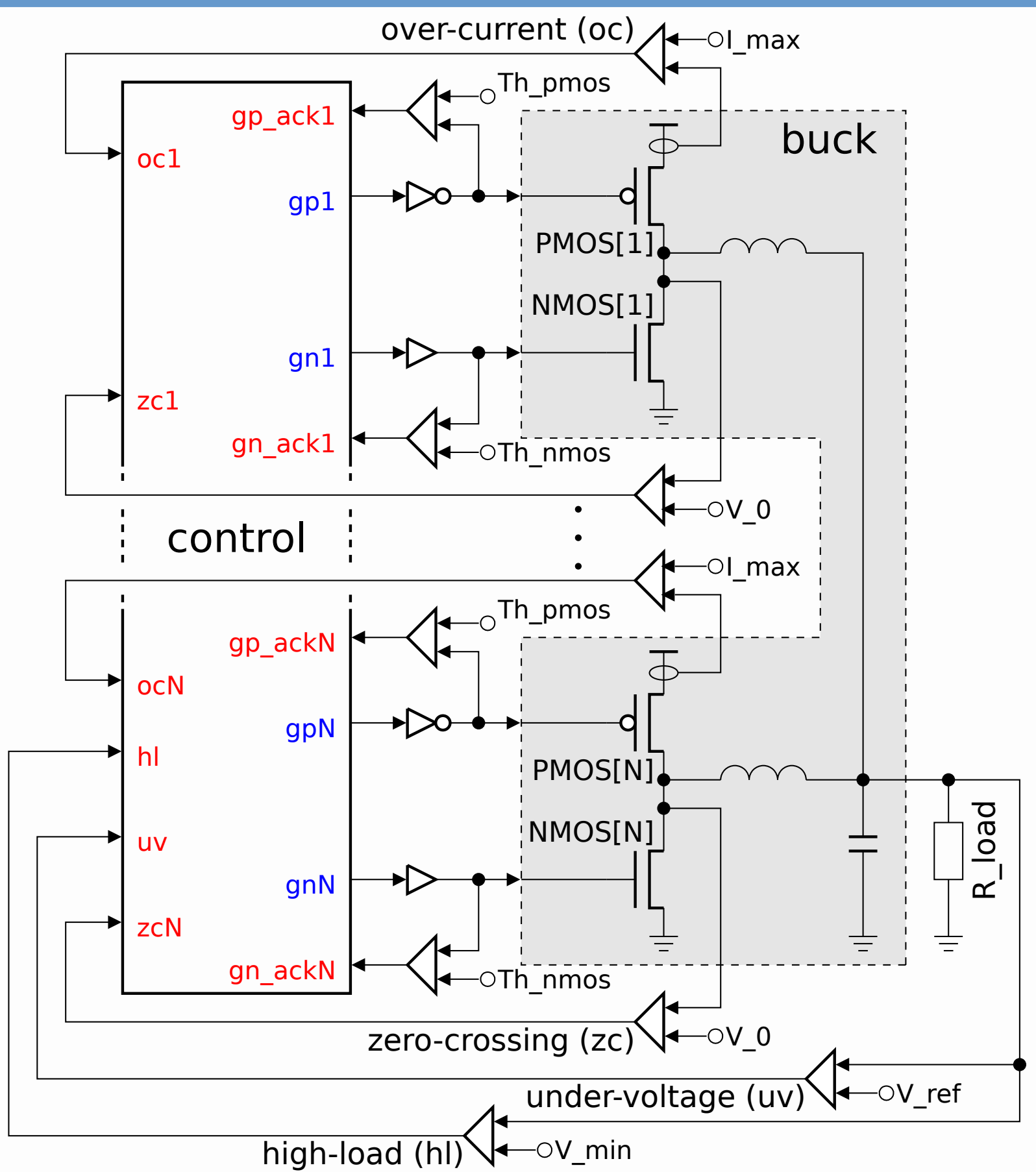


- Normal mode

- Phases are activated sequentially

- Phases may overlap

- High-load mode

- All phases are activated simultaneously

- Benefits

- Faster reaction to the power demand

- Heat dissipation from a larger area

- Decreased ripple of the output voltage

- Smaller transistors and coils 


\section{Synchronous design}

- Two clocks: phase activation $(\sim 5 \mathrm{MHz})$ and sampling $(\sim 100 \mathrm{MHz})$

(:) Easy to design (RTL synthesis flow)

(2) Response time is of the order of clock period

:) Power consumed even when idle

(2) Non-negligible probability of a synchronisation failure

- Manual ad hoc design to alleviate the disadvantages

(:) Verification by exhaustive simulation 


\section{Asynchronous design}

- Event-driven control decisions

(:) Prompt response (a delay of few gates)

(:) No dynamic power consumption when the buck is inactive

(:) Other well known advantages

(:) Insufficient methodology and tool support

- Our goals

- Formal specification of power control behaviour

- Reuse of existing synthesis methods

- Formal verification of the obtained circuits

- Demonstrate new advantages for power regulation (power efficiency, smaller coils, ripple and transient response) 


\section{High-level architecture: Token ring}

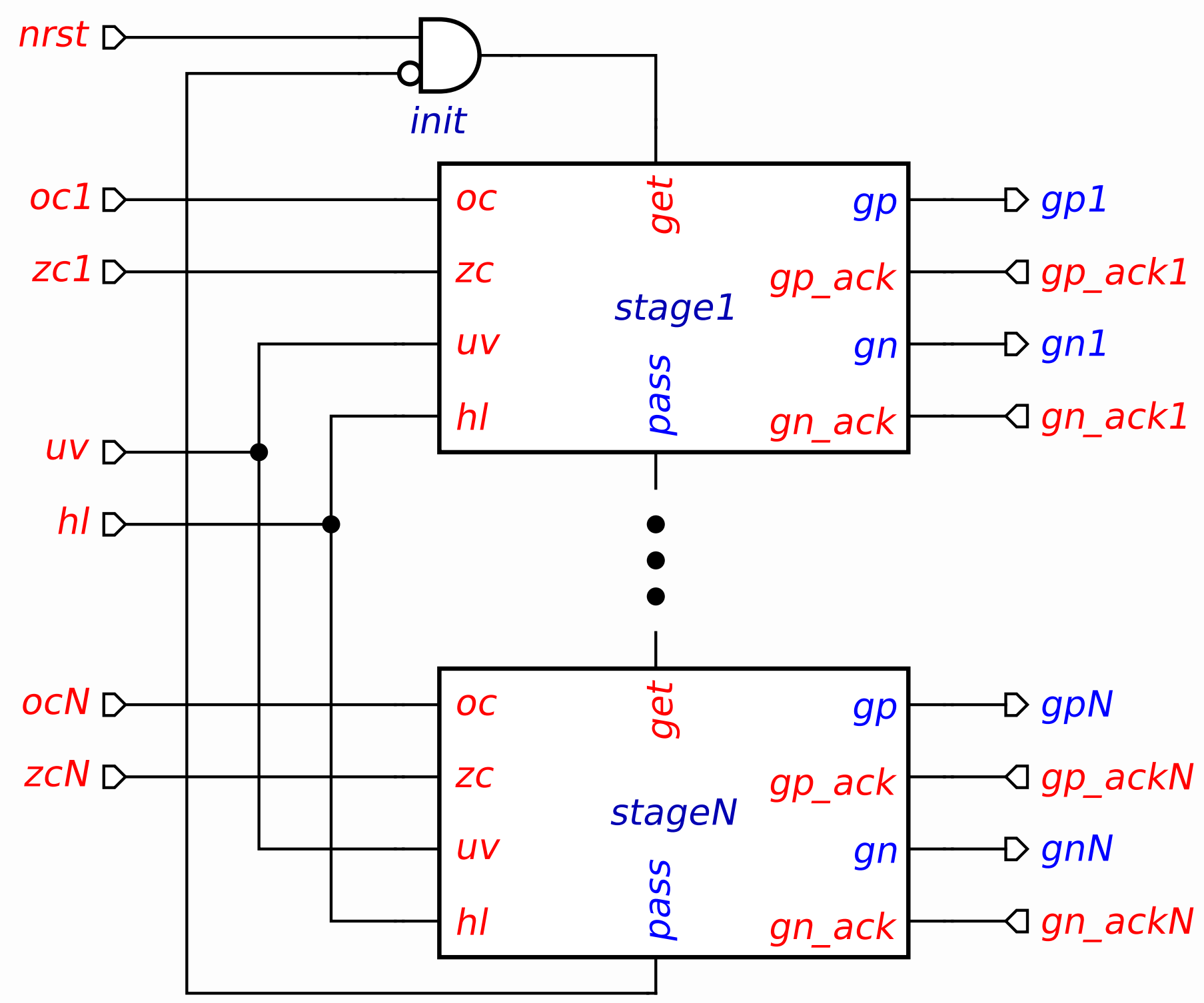




\section{High-level architecture: Stage}

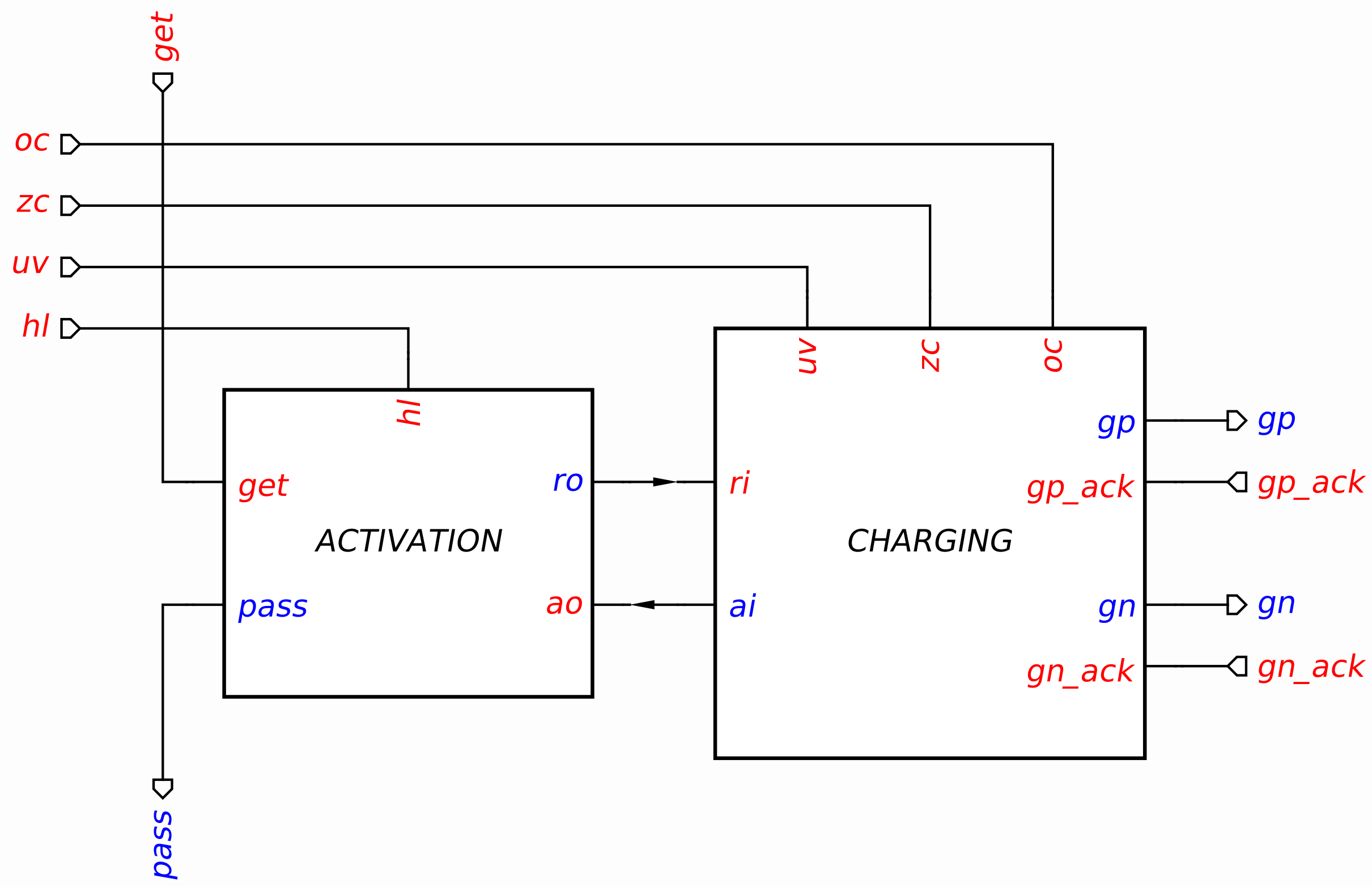




\section{High-level architecture: Activation}

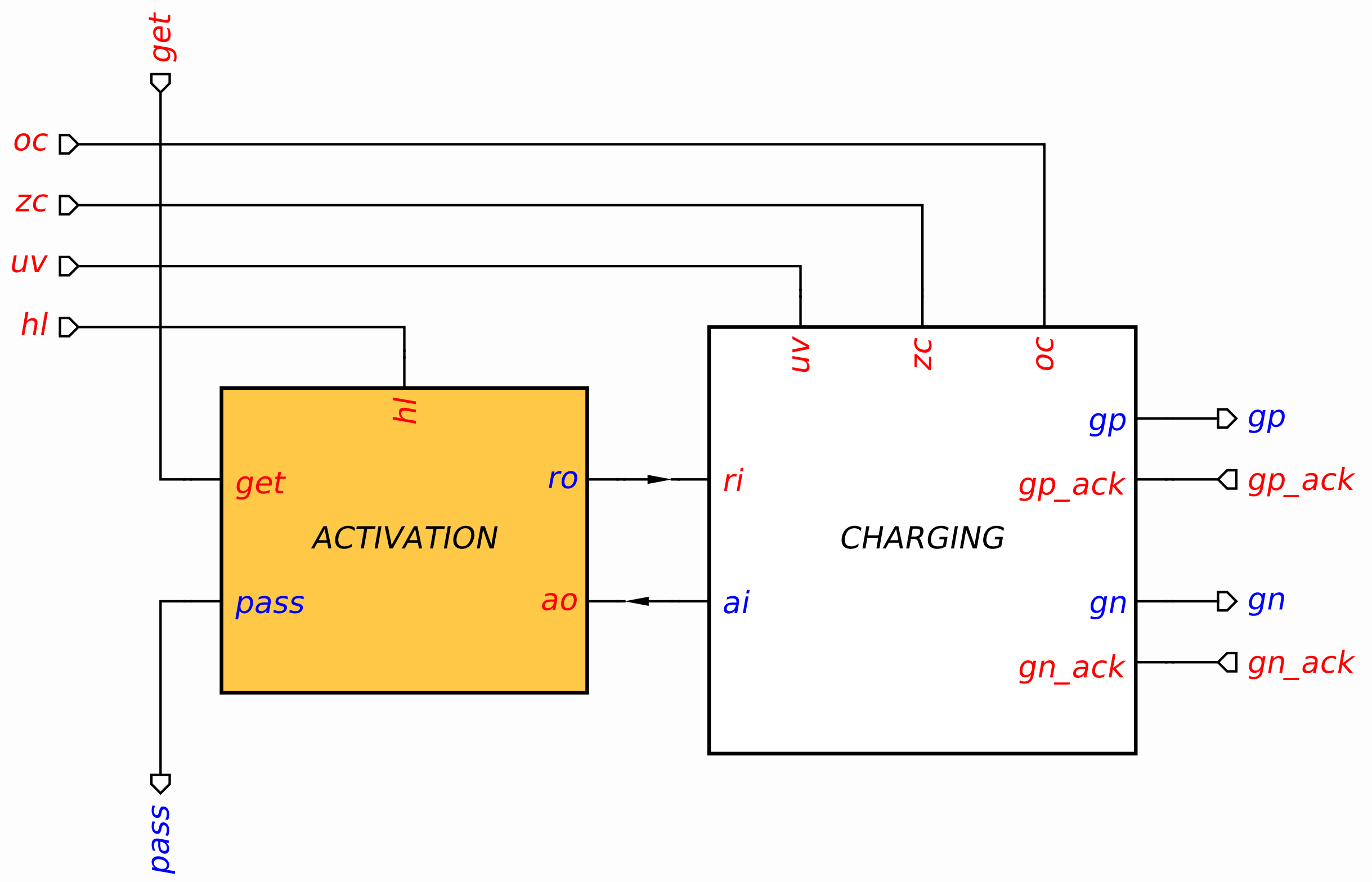




\section{High-level architecture: Activation}

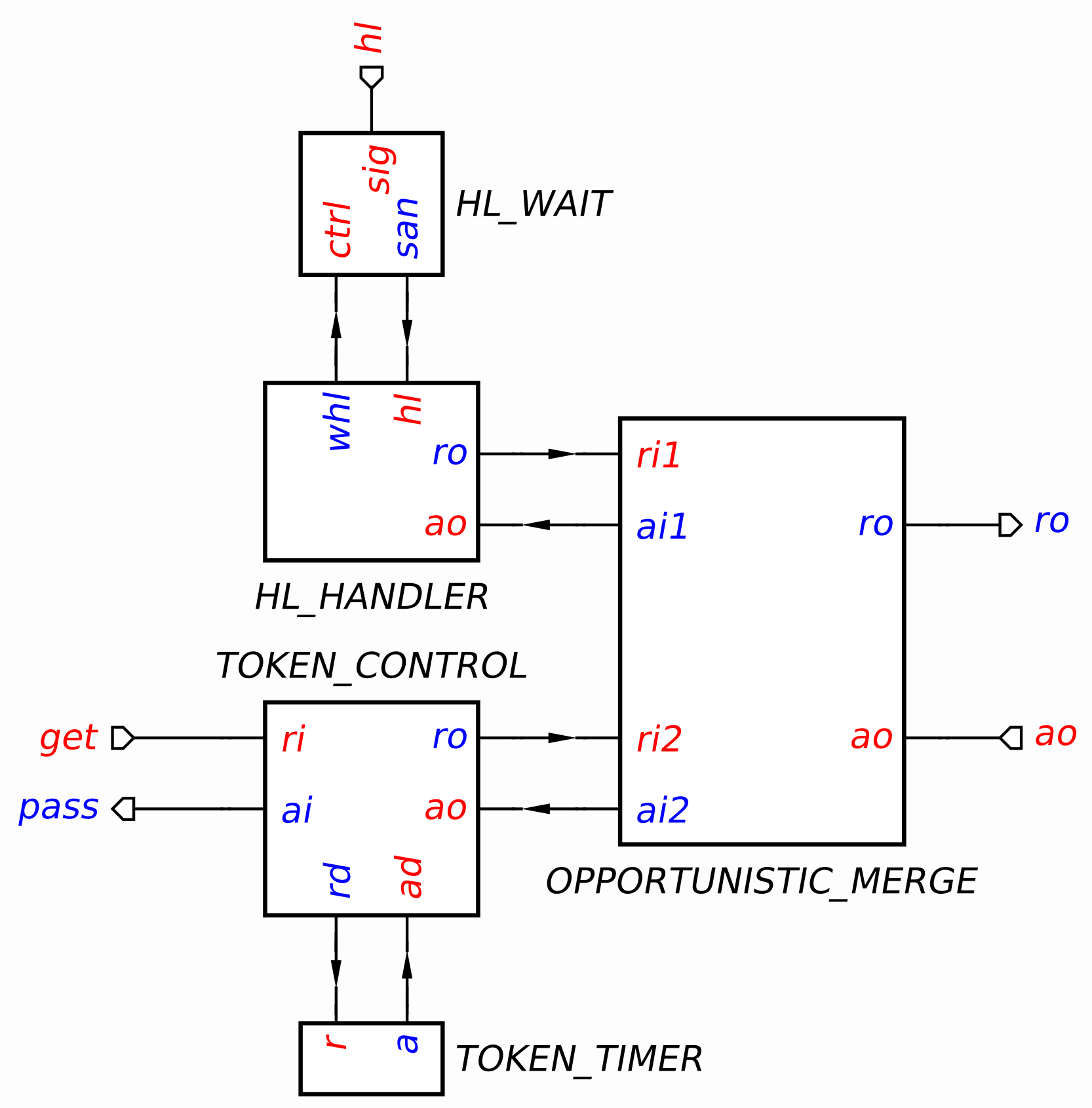




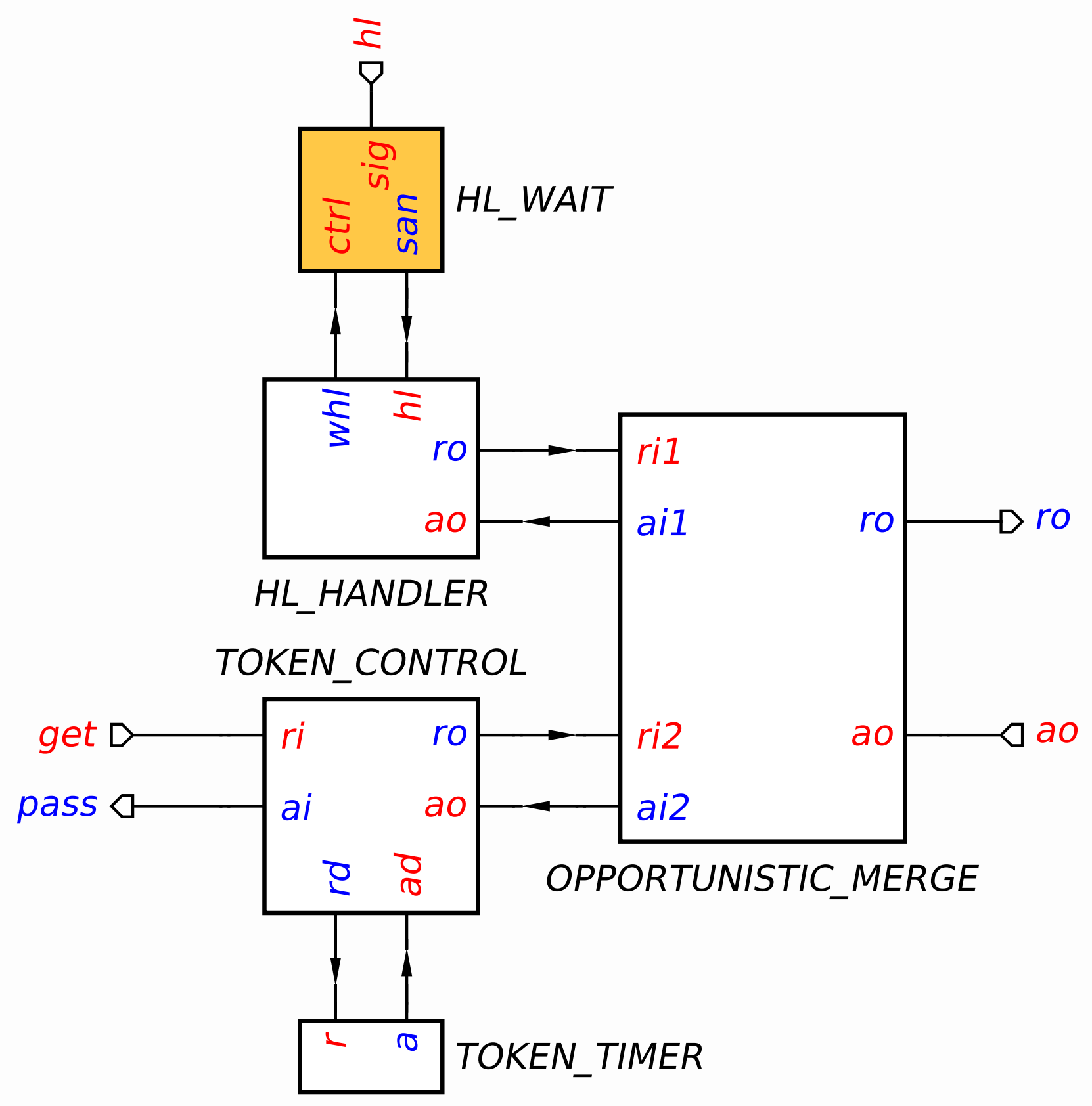




\section{WAIT element}

- STG specification

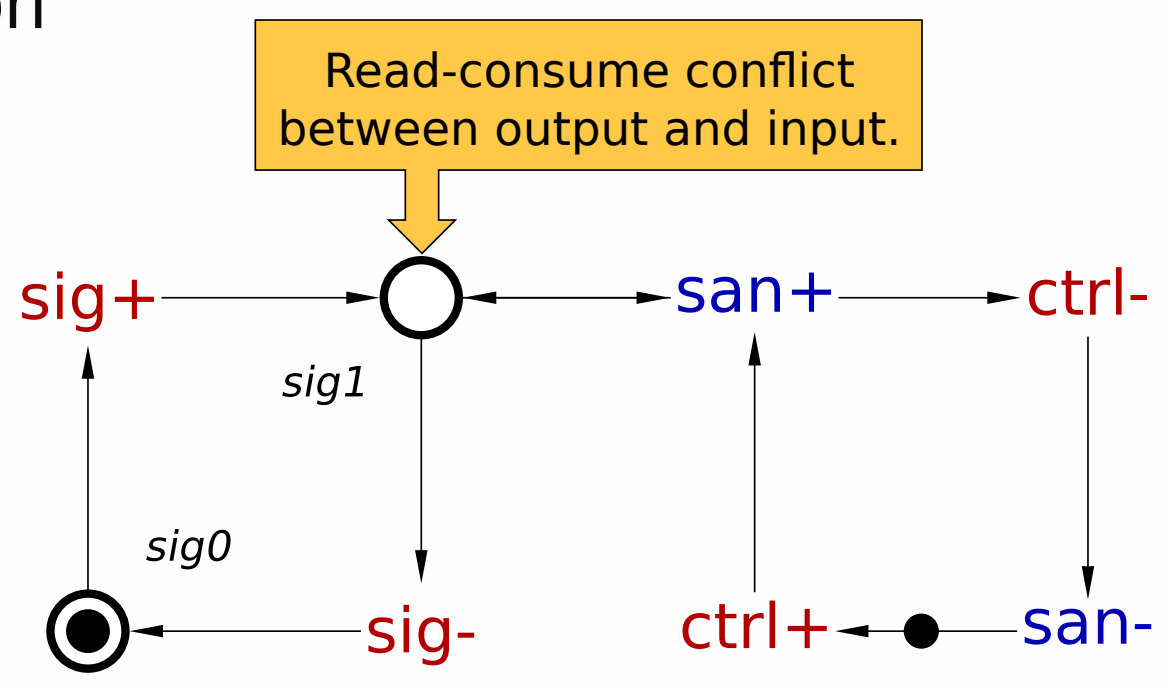

- ME-based solution

- Gate-level implementation
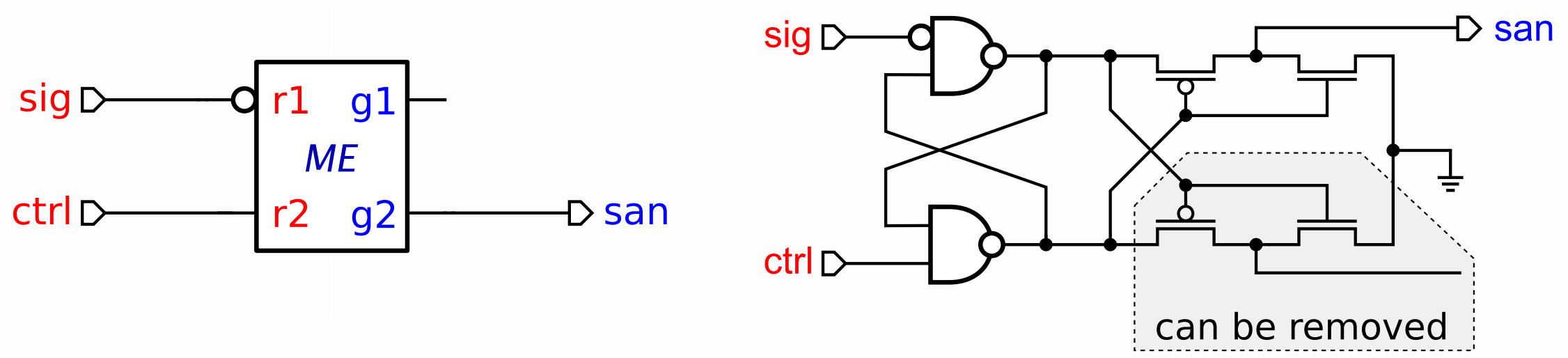


\section{High-level architecture: Charging}

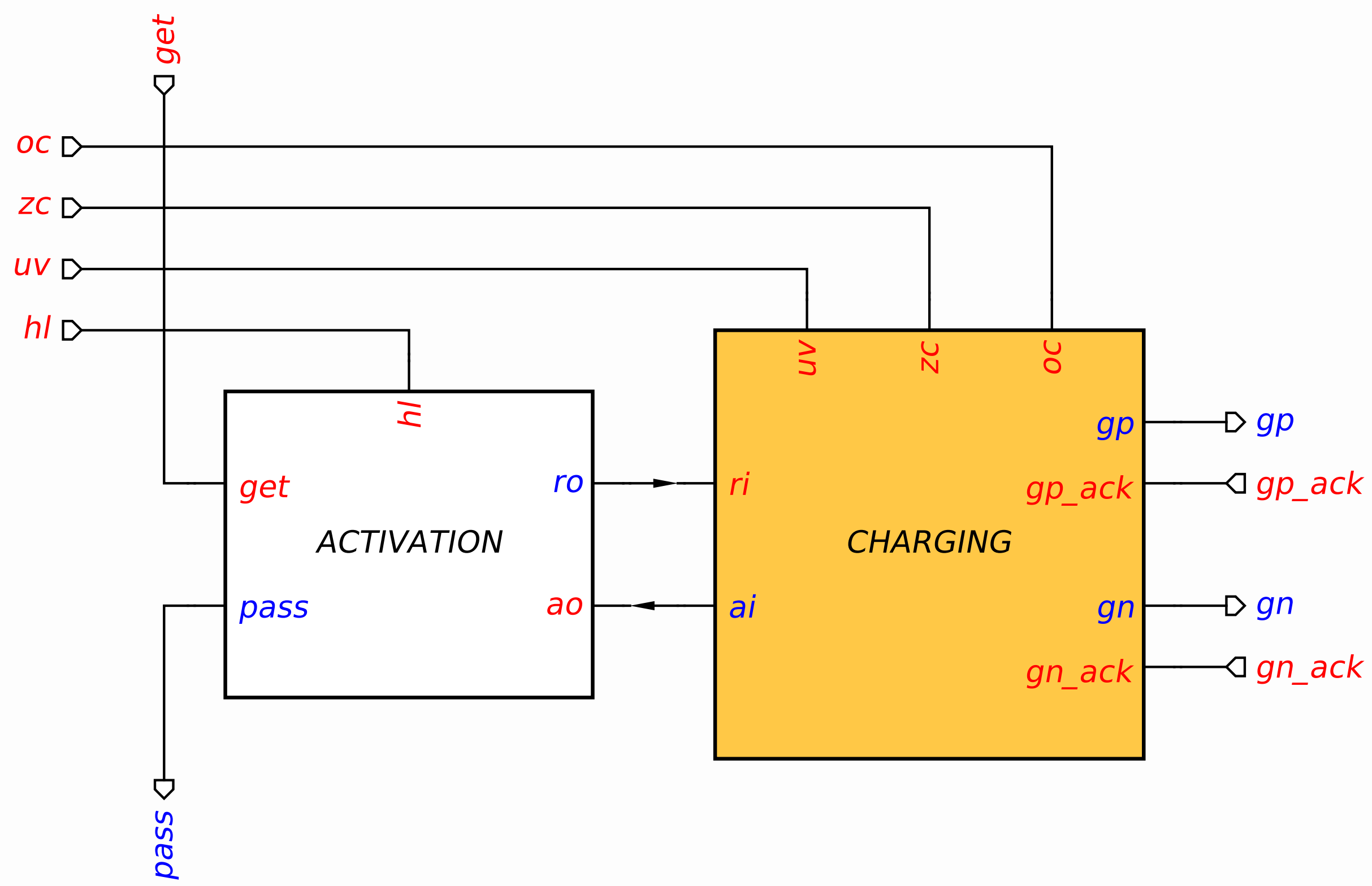




\section{High-level architecture: Charging}

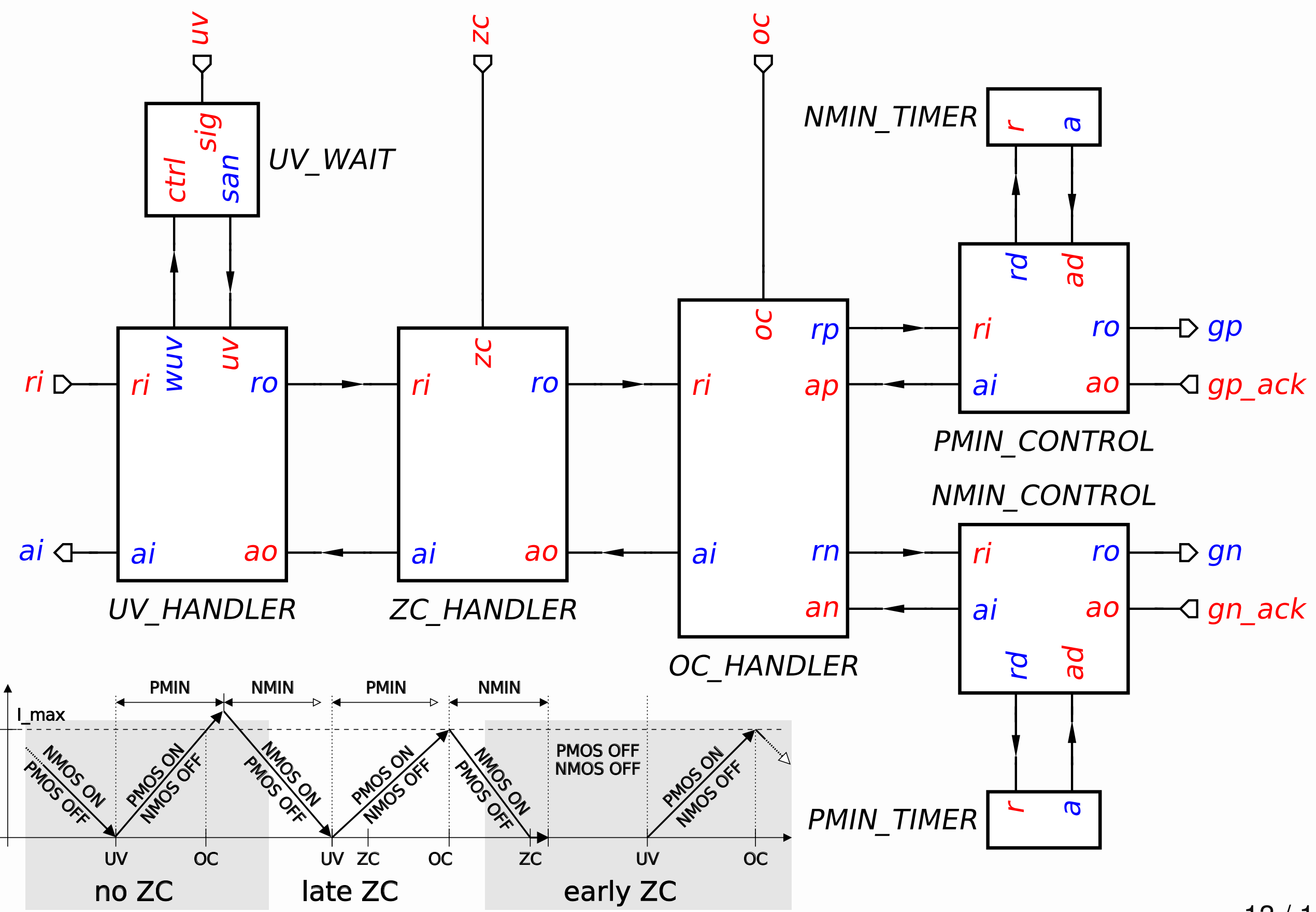


- Manual decomposition of the system into modules

- To create formal specification from informal requirements (feedback loop with engineers)

- To simplify specification and synthesis

- Some modules are reusable

- Some modules (Wait and Opportunistic Merge) are potential standard components

- Each component is specified using STGs

- Automatic synthesis into speed-independent circuits 


\section{ZC_HANDLER module}

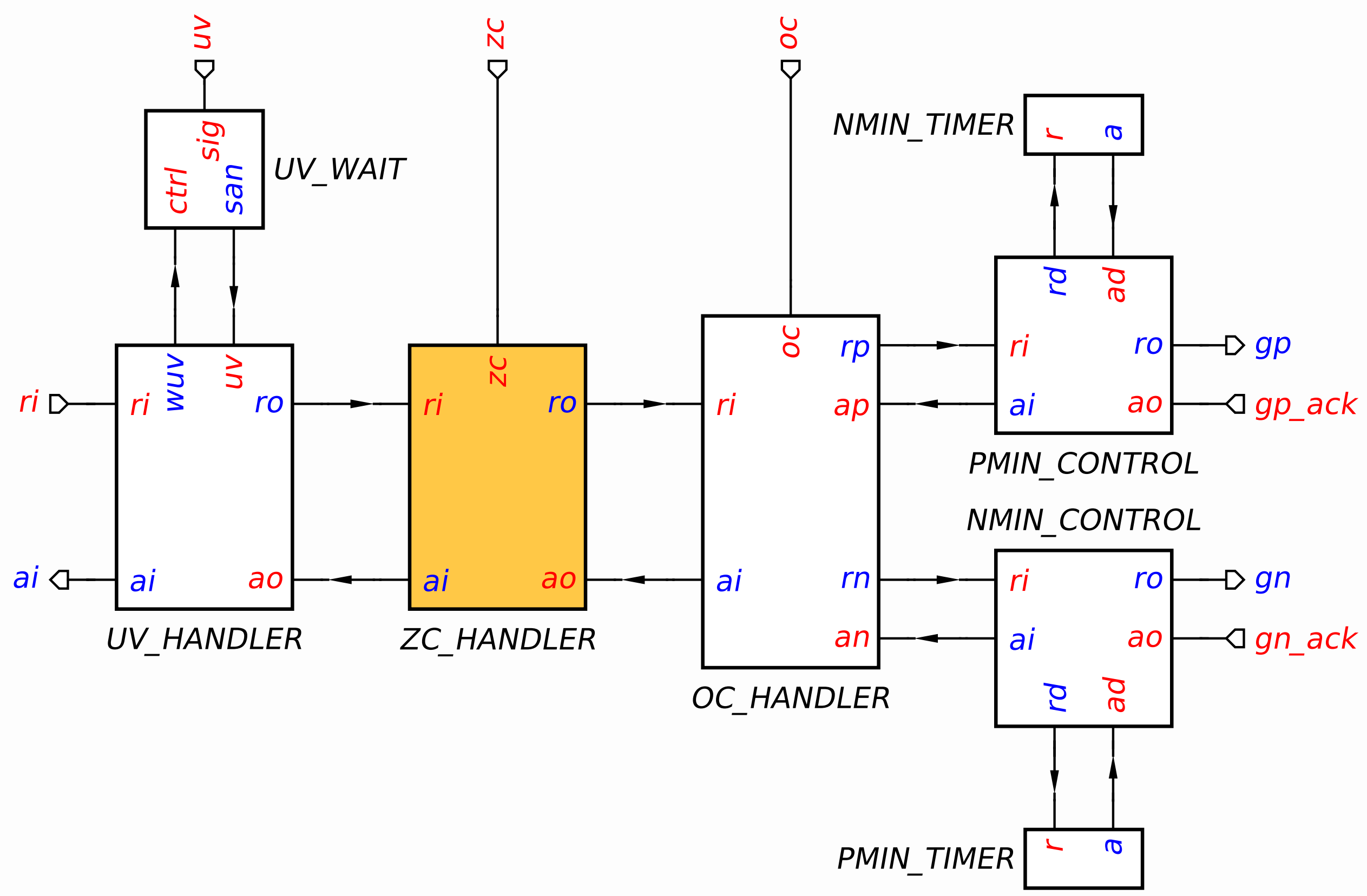




\section{ZC_HANDLER module}

- STG specification

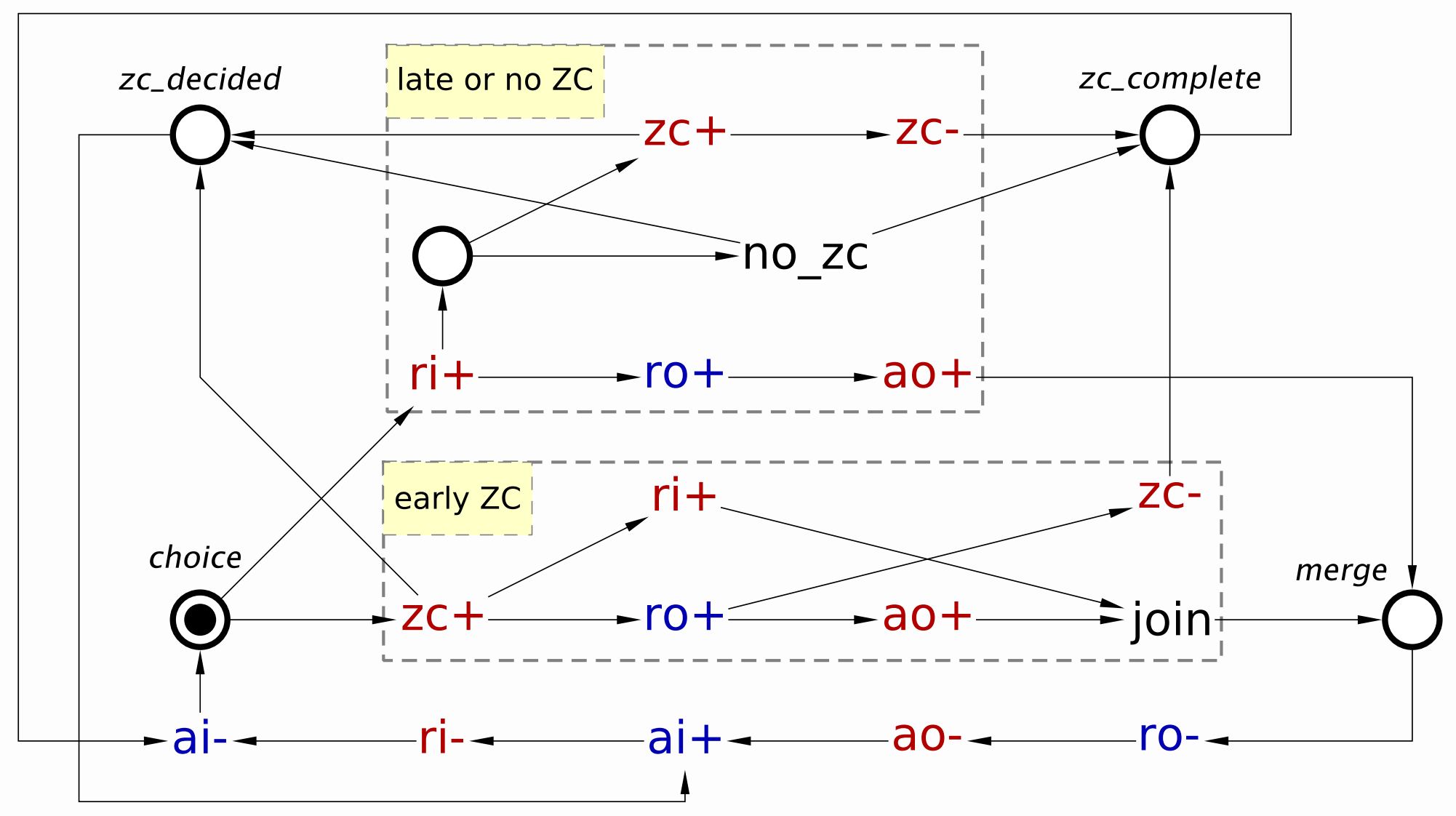




\section{ZC_HANDLER module}

- Speed-independent implementation

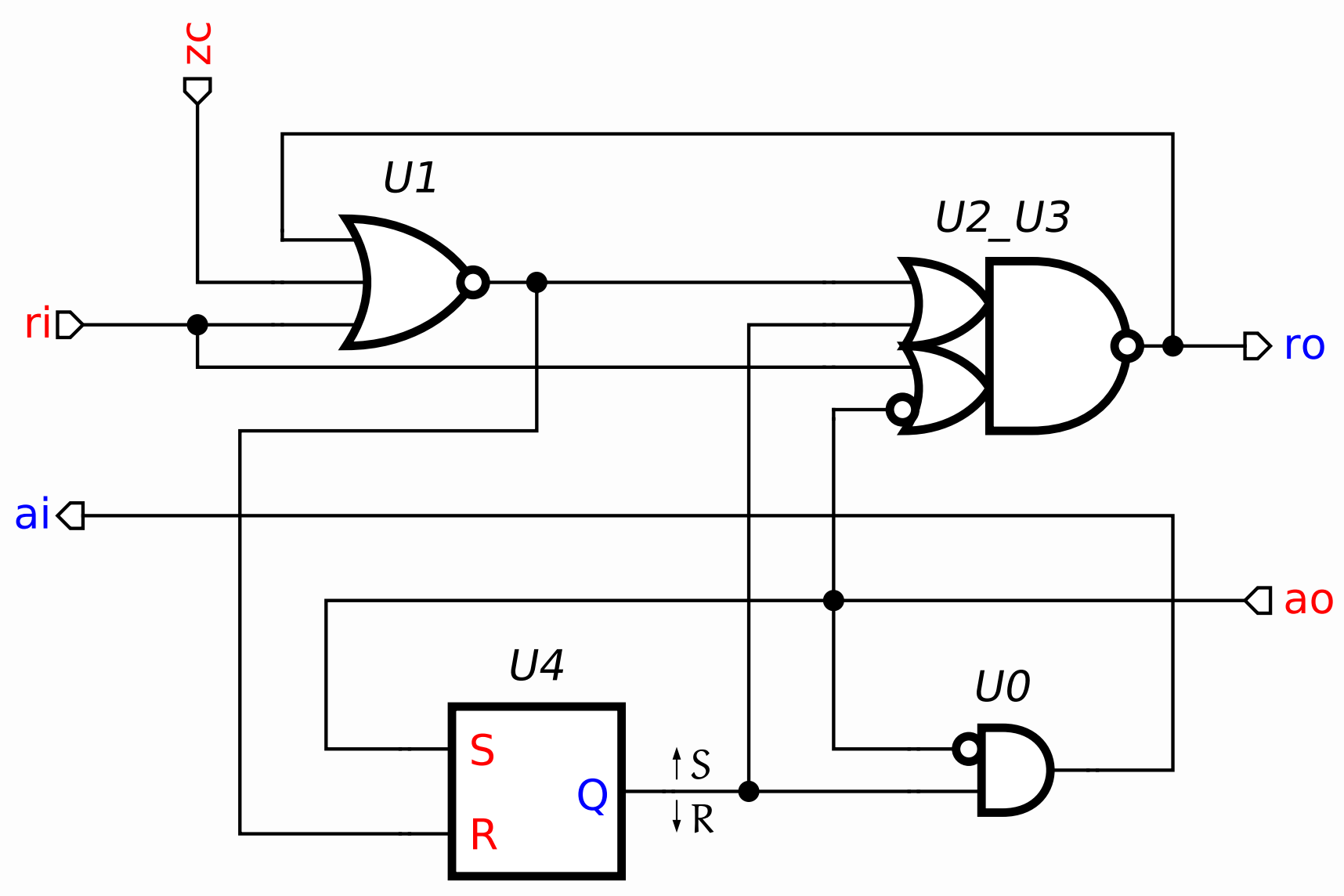




\section{Formal verification}

- STG verification

- All standard speed-independence properties

- PMOS and NMOS are never ON simultaneously

(no short circuit)

- Some timers are used in a mutually exclusive way and can be shared

- Circuit verification

- Conforms to the environment

- Deadlock-free and hazard-free under the given environment 


\section{Tool support: WORKCRAFT}

- Framework for interpreted graph models (STGs, circuits, FSMs, dataflow structures, etc.)

- Interoperability between models

- Elaborated GUI

- Includes many backend tools

- PETRIFY - STG and circuit synthesis, BDD-based

- PUNF - STG unfolder

- MPSAT - unfolding-based verification and synthesis

- PCOMP - parallel composition of STGs 


\section{Tool support: WORKCRAFT}

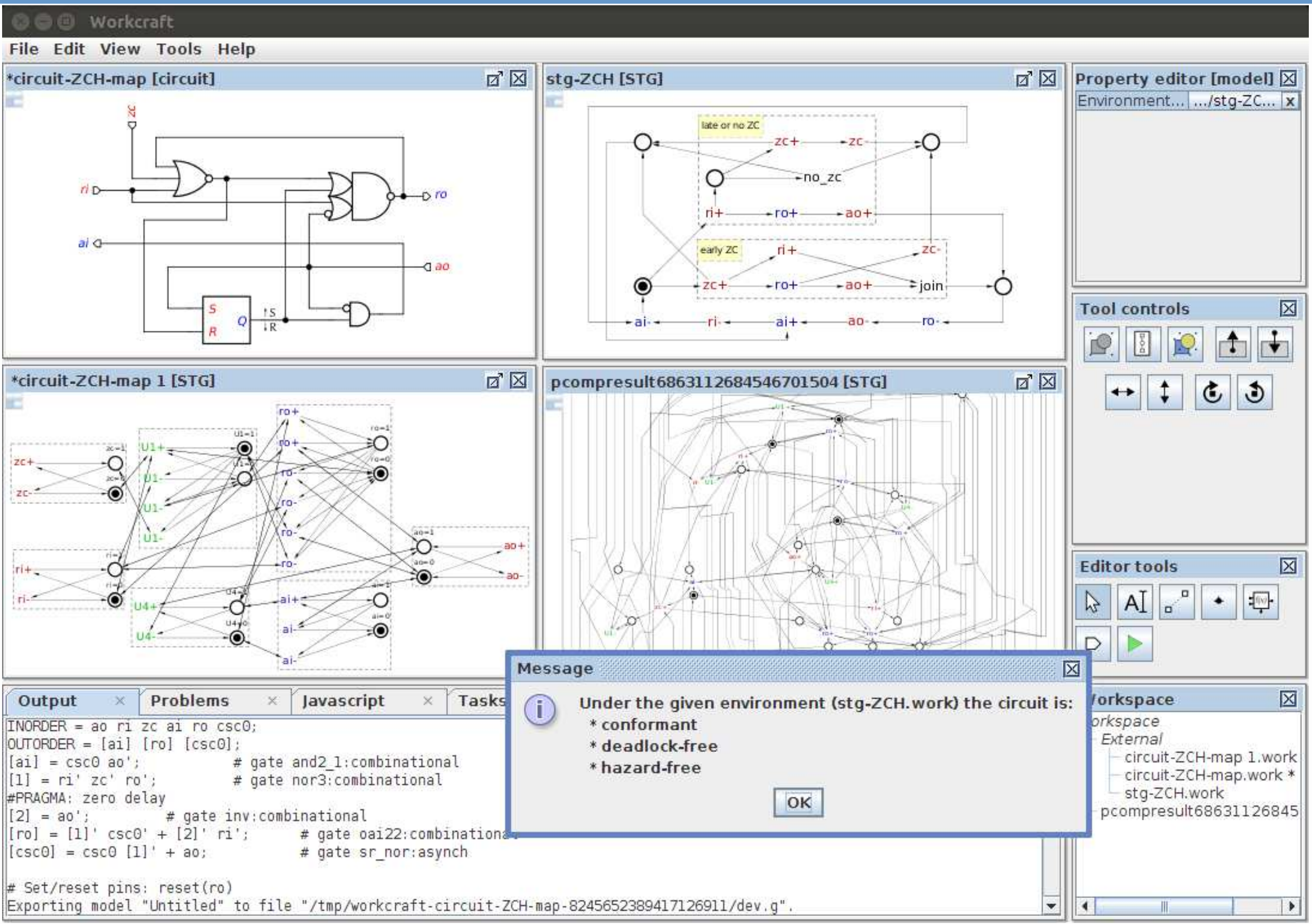


- Fully asynchronous design of multiphase buck controller

- Quick response time: few gate delays, all mutexes are outside the critical path

- Reliable: no synchronisation failures

- Design flow is automated to large extent

- Automatic logic synthesis

- Formal verification at the STG and circuit levels

- New standard components (Wait and Opportunistic Merge)

- Future work

- Measurements!

- Development of WORKCRAFT to support hierarchical design

- Co-simulation and co-verification of digital/analogue circuits

- Better integration with the Synopsys and Cadence flows 